\title{
Fuentes de luz de uso clínico en el pre grado: 7 años de seguimento
}

\author{
Light curing units in the undergraduate dental clinics: 7 years of follow-up
}

Terezinha Jesus Esteves Barata ${ }^{1, a}$, Igor Cesar Ribeiro de Carvalho ${ }^{1, b}$, Francisco Antonio Uchoa-Junior ${ }^{1, b}$, Tainah Costa Firmiano ${ }^{1, \mathrm{c}}$, Amanda Pedrosa Oliveira ${ }^{2, \mathrm{~d}}$, Gersinei Carlos de Freitas ${ }^{1, \mathrm{e}}$, Lawrence Gonzaga Lopes ${ }^{1, a}$, Érica Miranda de Torres ${ }^{1, \mathrm{f}}$

\section{RESUMEN}

Objetivo: Realizar, durante siete años ininterrumpidos, un control y seguimiento de las fuentes de luz disponibles en las clínicas odontológicas de pre graado de una Institución de Enseñanza Superior Pública Brasileña. Material y métodos: Durante el período comprendido entre 2011-2017 se evaluó el control de calidad de las fuentes de luz de la Facultad de Odontología de la Universidad Federal de Goiás a través del análisis de su estado de conservación y de su densidad de potencia $\left(\mathrm{mW} / \mathrm{cm}^{2}\right)$. Los datos obtenidos se sometieron a un análisis estadístico descritpivo y a las pruebas de Levene, Shapiro-Wilk, Kruskal-Wallis, Dunn y Chi-cuadrado $(\mathrm{P}<0,05)$. Resultados: No se observaron diferencias estadísticas significativas del estado de conservación de las fuentes de luz entre los años 2012, 2016 y 2017 (p>0,05). Sí se observaron diferencias estadísticas significativas entre los valores de densidad de potência entre dichos años $(\mathrm{p}<0,0001)$. En las comparaciones por pares, los valores de densidad de potencia para los años $2011(\mathrm{p}<0,01)$ y $2012(\mathrm{p}<0,05)$ fueron estadísticamente diferentes de los años 2015, 2016 y 2017. Conclusiones: Comparativamente se observó una mejora sustancial del estado de conservación de las fuentes de luz en los cuatro años iniciales de evaluaciones, con una disminución en el quinto año y una estabilización en los dos últimos años. Durante los siete años de estudio de la densidad de potencia de las fuentes de luz se observó en un aumento gradual de la misma con una estabilización de los resultados a partir del quinto año de control de calidad.

PALABRAS CLAVE: clínicas odontológicas, control de calidad, auto-curación de resinas dentales, luces de curación dental. 


\section{SUMMARY}

Objetive: To monitor the light curing units available at undergraduate dental clinics of a Brazilian Public Higher Education Institution for seven uninterrupted years. Materials and methods: In the period between 2011-2017, the quality control of light curing units at the Faculty of Dentistry, Federal University of Goiás, was evaluated by analyzing their state of conservation and power density $\left(\mathrm{mW} / \mathrm{cm}^{2}\right)$. Data obtained were subjected to descriptive statistical analysis and Levene, Shapiro-Wilk, Kruskal-Wallis, Dunn and Chi-square tests $(\mathrm{P}<0.05)$. Results: Regarding the conservation status of the light curing units, comparatively, there was no statistically significant difference between the years 2012, 2016 and 2017 ( $>>0.05$ ). Statistically significant differences were found for power density values between years $(\mathrm{p}<0.0001)$. In pairwise comparisons, the power density values for the years $2011(\mathrm{p}<0.01)$ and $2012(\mathrm{p}<0.05)$ were statistically different from the years 2015, 2016 and 2017. Conclusions: A significant improvement in the state of conservation of light curing units was observed in the initial four years of evaluations, with a decline in the fifth year and stabilization in the last two years. Regarding the power density of the light curing units, a gradual increase was observed in the seven years of study, with stabilization of the results after the fifth year of quality control.

KEYWORDS: dental clinics, quality control, polymerization, curing lights dental (DeCS).

\section{INTRODUÇÃO}

A Odontologia Adesiva consolidou-se a partir do vínculo direto entre os materiais dentários fotopolimerizáveis e as fontes de luz, também denominados de aparelhos fotopolimerizadores (1). Isto porque, esses materiais necessitam da emissão de energia luminosa para a conversão de monômeros presentes na sua composição de materiais resinosos em polímeros(1). E, por sua vez, as fontes de luz são os equipamentos odontológicos responsáveis pela fotopolimerização, ou seja, pela emissão da energia luminosa $(1,2,3)$.

Portanto, considerando-se que um número superior a quinhentos milhões de restaurações dentárias diretas foram realizadas em média, por ano no mundo, e destas, $55 \%$ utilizaram os sistemas adesivos e as resinas compostas (4). Infere-se, portanto, que o sucesso clínico das restaurações, esteja relacionado diretamente ao adequado funcionamento das fontes de luz $(3,5)$. Tal assertiva vincula-se ao fato de que a incompleta conversão da matriz polimérica dos materiais dentários fotopolimerizáveis, ocasionará a instabilidade química dos mesmos e, por conseguinte, aumento do teor de monômeros residuais não fotopolimerizados $(3,6)$. Além disso, a inadequada fotopolimerização, também está relacionada à formação de fendas ("gaps") na interface material dentário-estrutura dentária, uma vez que haverá comprometimento da resistência adesiva e das demais propriedades dos materiais dentários fotopolimerizáveis $(5,7,8,9)$. Então, a formação desse "gap" permitirá a degradação marginal e/ou infiltração, e consequentemente a recorrência de lesões cariosas, uma vez que haverá a migração de microrganismos por meio da interface dente - material dentário (1,3,5,7). Do mesmo modo, o comprometimento da resistência adesiva também poderá culminar no aumento da sensibilidade pósoperatória, bem como de manchamentos superficiais $(3,5,7)$. Logo, uma inadequada fotopolimerização comprometerá as propriedades dos materiais dentários, acarretando o insucesso do procedimento clínico realizado $(8,10,11)$.

É imperativo compreender, portanto, a cinética do processo de fotopolimerização e sua direta relação com a emissão de energia luminosa (3). Isto porque, a densidade, intensidade ou irradiância de potência, o tempo de exposição e a correlação entre o espectro de luz emitido pela fonte de luz e o espectro de absorção do seu fotoiniciador estão relacionadas integralmente às fontes de luz $(1,2,3)$.

Dentre esses fatores a densidade de potência mede a emissão de energia luminosa emitida pelas fontes de luz, ou seja, a potência das fontes de luz medida $\mathrm{em} \mathrm{mW} / \mathrm{cm}^{2}$ (12). Quando se associa a densidade de potência $\left(\mathrm{mW} / \mathrm{cm}^{2}\right)$ ao tempo de exposição da luz sobre o material dentário tem-se a densidade de energia que é calculada em $\mathrm{J} / \mathrm{cm}^{2}$ (12). Nesse momento, deve-se 
reportar ainda à diretriz $\mathrm{n}^{\circ} 48$ da ANSI/ADA (2004) concernente às fontes de luz (13). Por essa diretriz, a densidade mínima aceitável quando se utilizar as fontes de luz de Diodos Emissores de Luz (LED) foi definida em $600 \mathrm{~mW} / \mathrm{cm}^{2}$, pois o LED geralmente requer menor tempo exposição (13).

A partir da compreensão da cinética da fotopolimerização pode-se afirmar que as fontes de luz a influenciam de forma direta (10), seja positiva ou negativamente. Com base nessa argumentação, a presença de trincas, faturas e resíduos de materiais dentários fotopolimerizáveis, na ponta transmissora de luz são fatores negativos ao processo de fotopolimerização $(14,15,16)$. Dessa forma, as fontes de luz requerem manutenção e monitoramento periódicos a fim de garantirem a adequada fotopolimerização $(14,17)$.

Com base na relação direta entre a fotopolimerização e os procedimentos clínicos que utilizam materiais dentários fotopolimerizáveis, este estudo objetivou verificar e comparar o estado de conservação e a densidade de potência das fontes de luz disponíveis para o atendimento clínico em uma Instituição de Ensino Superior Pública Odontológica no Brasil, no período 2011 a 2017.

\section{MATERIAL E MÉTODOS}

As fontes de luz, em uso clínico na graduação, da Faculdade de Odontologia da Universidade Federal de Goiás, Instituição de Ensino Superior Pública, localizada no município de Goiânia, Goiás, Brasil, foram avaliadas, no período de 2011 a 2017, quanto ao estado de conservação e densidade de potência.

Quanto ao estado de conservação das fontes de luz analisou-se a presença de trincas, fraturas e/ou resíduos de materiais adesivos na ponta transmissora de luz, bem como danos físicos ao equipamento, de acordo com a metodologia proposta por Gonçalves et al., (17).

A verificação da densidade de potência de cada fonte de luz foi realizada com um radiômetro (Curing Radiometer Model 100 p/n - 10503, Demetron Research Corp., EUA), de acordo com o protocolo proposto por Marson, Mattos e Sensi (18) em mW/ $\mathrm{cm}^{2}$. Por este protocolo a ponta ativa da fonte de luz foi posicionada de maneira centralizada e perpendicular no radiômetro, e então três leituras foram realizadas, obtendo-se uma média aritmética, que foi usada na análise estatística. A primeira leitura era efetuada após 10 segundos do acionamento da fonte de luz, as leituras 2 e 3 foram realizadas de forma consecutivas com intervalos de 30 segundos entre as mesmas (18). Os dados foram submetidos à análise estatística por meio dos softwares SPSS 24 (Chicago, IL, EUA) e GraphPadInstat (La Jolla, EUA), ao nível de significância de 5\%. Os valores de densidade de potência foram avaliados quanto à homogeneidade das variâncias pelo teste Levene, e quanto à normalidade de distribuição por meio do teste Shapiro-Wilk. Por apresentarem ausência de normalidade e variâncias não homogêneas, os dados de densidade de potência foram comparados entre os anos por meio do teste não paramétrico Kruskal-Wallis com post hoc Dunn. Os dados de estado de conservação foram avaliados por meio do teste Qui-quadrado.

\section{RESULTADOS}

Diferenças estatisticamente significativas foram verificadas para os valores de densidade de potência entre os anos ( $<<0,0001$, teste Kruskal-Wallis). Nas comparações aos pares (teste post hoc Dunn), os valores de densidade de potência para os anos 2011 $(p<0,01)$ e $2012(p<0,05)$ foram estatisticamente diferentes dos anos 2015, 2016 e 2017. As demais comparações aos pares foram estatisticamente semelhantes $(p>0,05)$. A tabela 1 apresenta estatísticas descritivas para densidade de potência, representadas graficamente no Box-plot (Gráfico 1). A tabela 2

Tabela 1. Mediana, intervalo inter-quartil (Q25-Q75) e valores mínimo e máximo (Mín-Máx) para os dados de densidade de potência $\left(\mathrm{mW} / \mathrm{cm}^{2}\right)$ nos diferentes anos. Letras maiúsculas na coluna indicam semelhanças estatísticas ( $p>0,05$, teste Kruskal-Wallis, post hoc Dunn).

\begin{tabular}{llll}
\hline Anos & Mediana & Q25-Q75 & Mín-Máx \\
\hline 2011 & $215^{\mathrm{B}}$ & $143,7-421,2$ & $50-625$ \\
2012 & $271^{\mathrm{B}}$ & $200-458,7$ & $70-603$ \\
2013 & $406^{\mathrm{AB}}$ & $184,5-491,5$ & $100-796$ \\
2014 & $430^{\mathrm{AB}}$ & $250-750$ & $90-1000$ \\
2015 & $600^{\mathrm{A}}$ & $293,7-820,5$ & $136-1933$ \\
2016 & $550^{\mathrm{A}}$ & $375-700$ & $100-1900$ \\
2017 & $533^{\mathrm{A}}$ & $433-900$ & $166-1750$ \\
\hline
\end{tabular}




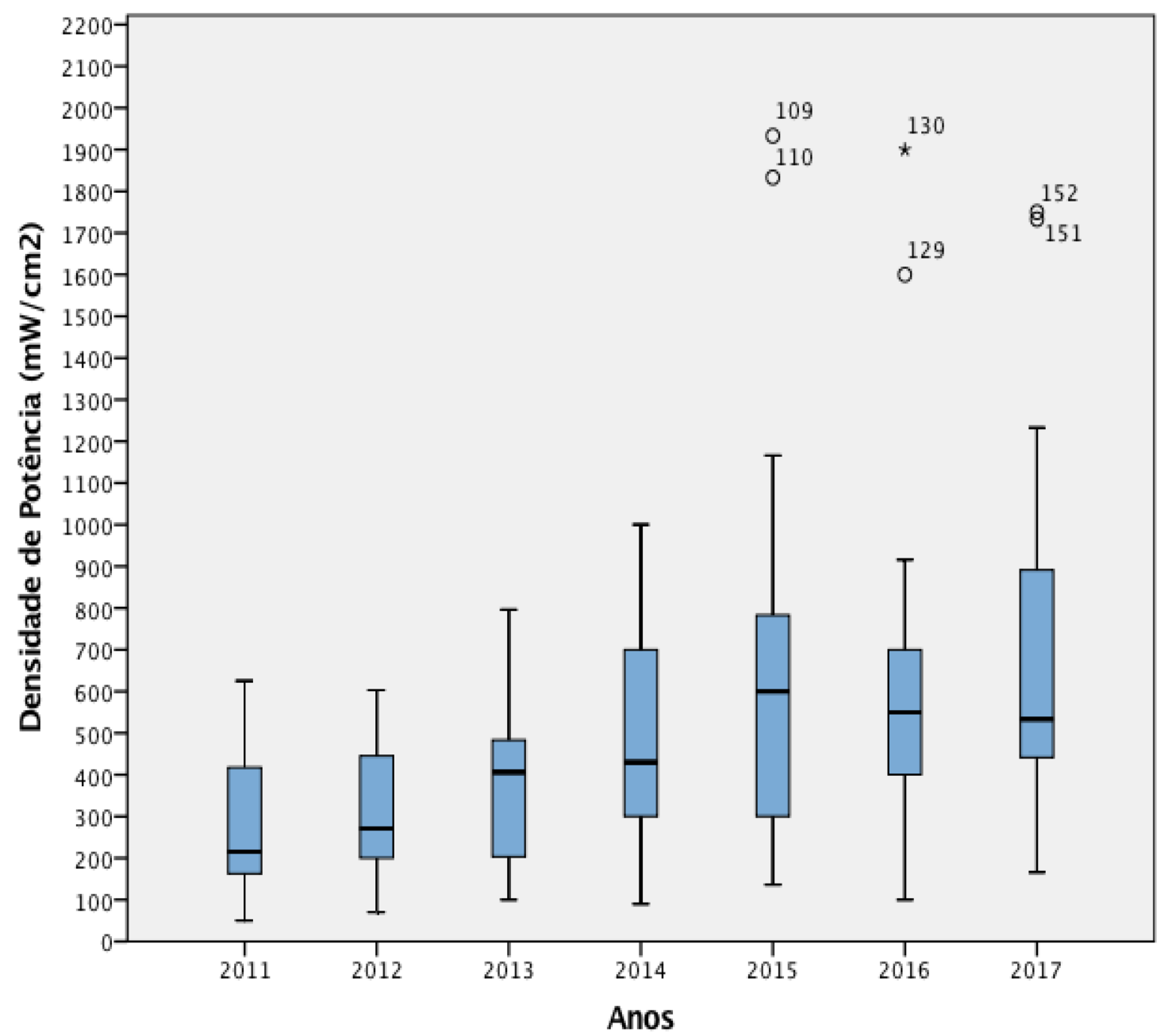

Gráfico 1. Box-plot dos valores de densidade de potência $\left(\mathrm{mW} / \mathrm{cm}^{2}\right)$ nos diferentes anos.

Tabela 2. Frequências absolutas (percentuais) para os dados de estado de conservação nos diferentes anos. Letras maiúsculas iguais indicam semelhanças estatísticas nas colunas, para comparações entre os anos; letras minúsculas iguais indicam semelhanças estatísticas nas linhas, para comparações dentro de cada ano ( $>0,05$, teste Quiquadrado).

\begin{tabular}{lll}
\hline \multirow{2}{*}{ Anos } & \multicolumn{2}{l}{ Estado de Conservação } \\
\cline { 2 - 3 } & Adequado & Inadequado \\
\hline 2011 & $2(10 \%) \mathrm{Aa}$ & $18(90 \%) \mathrm{Ab}$ \\
2012 & $10(45,5 \%) \mathrm{ABa}$ & $12(54,5 \%) \mathrm{ABa}$ \\
2013 & $13(61,9 \%) \mathrm{Ba}$ & $8(38,1 \%) \mathrm{Ba}$ \\
2014 & $25(75,8 \%) \mathrm{Ba}$ & $8(24,2 \%) \mathrm{Bb}$ \\
2015 & $13(59,1 \%) \mathrm{Ba}$ & $9(40,9 \%) \mathrm{Ba}$ \\
2016 & $10(47,6 \%) \mathrm{ABa}$ & $11(52,4 \%) \mathrm{ABa}$ \\
2017 & $10(52,6 \%) \mathrm{ABa}$ & $9(47,4 \%) \mathrm{ABa}$ \\
\hline
\end{tabular}

apresenta estatísticas descritivas para a variável estado de conservação, bem como resultados obtidos com o teste Qui-quadrado.

\section{DISCUSSÃO}

Comparativamente, à literatura correlata analisada, até o primeiro trimestre de 2020 (7, 9, $11,14-16,18-26,28-32)$, o presente estudo tornouse, o mais longo controle de qualidade de fontes de luz, uma vez que o fez anualmente, por sete anos de forma ininterrupta. Esse controle revelou uma melhoria no estado de conservação das fontes de luz até o quarto ano da avaliação (2014), com declínio e estabilização a partir de 2015 até 2017. Entre 2011 e 2014 as fontes de luz não passíveis de manutenção foram substituídas por novos equipamentos, todavia 
nos anos seguintes (2015-2017) nenhuma nova fonte de luz foi adquirida, a fim de substituir àquelas não passíveis de manutenção. Isto justifica a estabilização das condições de conservação das fontes de luz observadas, no presente estudo.

Dentre os principais fatores que interferiram negativamente no estado de conservação na ponta transmissora de luz, a presente avaliação revelou a presença de resíduos de resina composta e/ou adesivo e/ou outro material dentário fotopolimerizável, bem como a presença de trincas e/ou fraturas. O estudo de Barghi, Fischer e Pham (16) constatou a presença de $75,8 \%$ de resíduos nas pontas transmissoras de luz, porém em consultórios odontológicos particulares. Estudo similar observou que $52,9 \%$ das pontas transmissoras das fontes de luz de Instituição de Ensino, Centro de Saúde Odontológico Público e consultórios particulares existentes na cidade de Sivas-Turquia apresentavam resíduos (9).

Concentrando-se a discussão nos resultados do presente estudo, realizado em Clínicas Odontológicas para o ensino de graduação, observou-se que $90 \%$ das fontes de luz disponíveis encontravam-se em inadequado estado de conservação na primeira avaliação realizada, em 2011. De forma similar Beltrani et al. (19) observaram, também, em âmbito acadêmico que $91,7 \%$ das pontas transmissoras de suas fontes de luz apresentaram a presença de detritos e 16,7\% exibiram algum tipo de fratura. Já o estudo de Nassar, Ajaj e Hasanain (15) revelou que 75\% fontes de luz disponíveis na sua Instituição de Ensino continham resíduos nas pontas transmissoras de luz e metade dessas fontes estavam alocadas nas clínicas para a graduação.

Ainda analisando esse mesmo critério, em ambiente universitário, porém em dois momentos distintos, observou-se inadequado estado de conservação das fontes de luz em 56,25\% e 69,23\% das amostras, com seis meses de intervalo entre as avaliações (24). No presente estudo, pode-se conjecturar que a inclusão no processo ensino-aprendizagem da graduação, de conteúdos teórico-práticos específicos relacionados a importância do controle de qualidade das fontes de luz e sua relação com o sucesso clínico foi benéfica. Esta pode ser uma das possíveis razões a serem atribuídas a melhora expressiva no estado de conservação das fontes de luz, no presente estudo. Visto que, $10 \%$ das fontes de luz na primeira e 75,8\% na quarta avaliação encontravam-se com adequado estado de conservação. Esses resultados corroboram o trabalho de Federlin e Price (33).

Então, os resultados do presente estudo respaldam a seguinte afirmativa: as fontes de luz com inadequado estado de conservação apresentam menor densidade de potência, por conseguinte inadequada fotopolimerização $\quad(7,9,10,11,14-$ $16,18,20,22,24,26,27,29,30,32)$. Os valores de densidade de potência (2011-2017), no presente estudo, revelaram um crescente aumento da primeira à quinta avaliações (Tabela 1). Na primeira e segunda avaliações, valores de densidade de potência menores do que $300 \mathrm{~mW} / \mathrm{cm}^{2}$ foram registrados, o que demonstrou uma situação grave em termos de inadequada fotopolimerização. A melhoria da densidade de potência, provavelmente, foi devido a aquisição de novas fontes de luz aliado com uso consciente das mesmas e o programa de manutenção preventiva. No período entre 2015 - 2017 (quinta a sétima avaliações) ocorreu uma estabilização da densidade de potência (533 e $600 \mathrm{~mW} / \mathrm{cm}^{2}$ ). Devese acrescentar que a partir da sexta avaliação (2016) as fontes de luz passaram a ser adquiridas pelos graduandos, o que levou em 2017 ao encerramento desse estudo. As fontes de luz ainda existentes continuam a ser analisadas e passam anualmente por manutenção preventiva, porém uma vez descartadas não são substituídas pela Instituição. Entretanto, o processo ensino-aprendizagem implementado continua a ocorrer de modo ininterrupto a partir dos resultados obtidos na primeira avaliação.

Em termos de comparação entre os resultados obtidos em Instituições Púbicas de Ensino no Brasil, observou-se que $32 \%$ e $26,31 \%$ das fontes de luz avaliadas apresentavam densidade de potência $\geq 400$ $\mathrm{mW} / \mathrm{cm}^{2}$ e inferior $100 \mathrm{~mW} / \mathrm{cm}^{2}$, respectivamente, na região Nordeste (20). Na região Sul a avaliação foi realizada em dois momentos distintos, com intervalo de seis meses entre os mesmos (24). A densidade de potência constatada foi inferior a $300 \mathrm{~mW} / \mathrm{cm}^{2}$ para $50 \%$ e $53,85 \%$ das fontes de luz na primeira e segunda avaliações, por essa ordem (24). Quanto aos resultados, na região Centro-Oeste, o presente estudo observou um expressivo aumento da densidade de potência entre a primeira $\left(143,7-421,2 \mathrm{~mW} / \mathrm{cm}^{2}\right)$ e a última (433 - $\left.900 \mathrm{~mW} / \mathrm{cm}^{2}\right)$ avaliações realizadas. Os 
primeiros resultados assemelham-se aos obtidos nas Instituições Públicas brasileiras das regiões Nordeste (20) e Sul (24). Não obstante, a densidade de potência registrada entre 2015 - 2017, no presente estudo variou entre $533-600 \mathrm{~mW} / \mathrm{cm}^{2}$. Tal resultado ainda é inferior $\left(921,6 \mathrm{~mW} / \mathrm{cm}^{2}\right)$ ao verificado nas clínicas de graduação na Arábia Saudita, em 2018 (15), porém nota-se uma expressiva melhora comparativamente aos anos anteriores do presente estudo e aos resultados obtidos nas regiões Nordeste(20) e Sul (24) do Brasil. Considerando os resultados obtidos no presente estudo e a literatura correlata $(7,11,14-18,22,23,25$ 32 ), pode-se afirmar com segurança que o controle de qualidade das fontes de luz é imperativo $(17,34)$. Conclui-se, portanto, que entre 2011 e 2014 observouse uma melhoria expressiva do estado de conservação das fontes de luz, com declínio em 2015 e estabilização em 2016 e 2017. O estado de conservação das fontes de luz influenciou na densidade de potência das mesmas. A densidade de potência das fontes de luz nos sete anos de avaliação do estudo foi ascendente com estabilização dos resultados a partir do quinto ano de controle de qualidade.

\section{Correspondencia:}

Terezinha Jesus Esteves Barata

Universidade Federal de Goiás, Faculdade de Odontologia.

Praça Universitária, s/n, Setor Universitário, GoiâniaGO, 74605-220.

Correo electrónico: terezinhabarata@ufg.br

\section{REFERÊNCIAS BIBLIOGRÁFICAS}

1. Cadenaro M, Maravic T, Comba A, et al . The role of polymerization in adhesive dentistry. Dent Mater. 2019;35(1):e1-e22.

2. Rueggeberg FA, Giannini M, Arrais CAG, Price RBT. Light curing in dentistry and clinical implications: a literature review. Braz Oral Res. 2017;31(suppl 1):e61.

3. Krämer N, Lohbauer U, García-Godoy F, Frankenberger R. Light curing of resin-based composites in the LED era. Am J Dent. 2008;21(3):135-42.

4. Heintze SD, Rousson V. Clinical effectiveness of direct class II restorations - a meta-analysis. J Adhes Dent. 201214(5):407-31.

5. Besegato JF, Jussiani EI, Andrello AC, et al. Effect of light-curing protocols on the mechanical behavior of bulk-fill resin composites. J Mech Behav Biomed Mater. 2019; 90:381-387.

6. Małkiewicz K, Wychowański P, Olkowska-
Truchanowicz J, et al. Uncompleted polymerization and cytotoxicity of dental restorative materials as potential health risk factors. Ann Agric Environ Med. 2017; 24(4):618-623.

7. Alquria T, Al Gady M, Khabeer A, Ali S. Types of polymerisation units and their intensity output in private dental clinics of twin cities in eastern province, KSA; a pilot study. J Taibah Univ Med Sci. 2018;14(1):47-51.

8. Hasslen JA, Barkmeier WW, Shaddy RS, Little JR. Depth of cure of high-viscosity bulk-fill and conventional resin composites using varying irradiance exposures with a light-emitting diode curing unit. J Oral Sci. 2019;61(3):425-30.

9. Eren D, Tutkan F. Investigation of the reliability of light-curing units in Sivas City, Turkey. Niger J Clin Pract. 2019;22(4):469-77.

10. Shimokawa CAK, Turbino ML, Giannini M, Braga RR, Price RB. Effect of light curing units on the polymerization of bulk fill resin-based composites. Dent Mater. 2018; 34(8):1211-21.

11. Al Shaafi M, Maawadh A, Al Qahtani M. Evaluation of light intensity output of QTH and LED curing devices in various governmental health institutions. Oper Dent. 2011;36(4):356-61.

12. Anusavice KJ, Shen C, Rawls HR. Compósitos de base resinosa (resina composta). In: Anusavice KJ, Shen C, Rawls HR. Phillips Materiais Dentários. Rio de Janeiro: Elsevier; 2013. p. 287-91.

13. American Dental Association. ANSI/ADA Standard No. 48 for Visible Light Curing Units - ADA48-2004. American Dental Association.; 2005.

14. Gonçalves LS, Gonçalves MB, Martins MM, Freitas GC, Lopes LG, Barata TJE. Clinical effectiveness of light-curing units of the School of Dentistry of the Federal University of Goias. RSBO. 2013; 10(3): 22833.

15. Nassar HM, Ajaj R, Hasanain F. Efficiency of light curing units in a government dental school. J Oral Sci. 2018;60(1):142-46.

16. Barghi N, Fischer DE, Pham T. Revisiting the intensity output of curing lights in private dental offices. Compend Contin Educ Dent. 2007;28(7):380-4.

17. Kopperud SE, Rukke HV, Kopperud HM, Bruzell EM. Light curing procedures -performance, knowledge level and safety awareness among dentists. J Dent. 2017;58:67-73.

18. Marson FC, Mattos, R, Sensi LG. Avaliação das condições de uso dos fotopolimerizadores. Revista Dentística on line. 2010; 9(19): 15-20.

19. Beltrani FC, Caldarelli PG, Kossatz S, Hoepner RG. Avaliação da intensidade de luz e dos componentes dos aparelhos fotopolimerizadores da Clínica Odontológica da Universidade Estadual de Londrina. Rev bras pesqui saúde, 2012; 14(1): 5-11. 
20. Borges FMGS, Rodrigues CC, Freitas SAA, Costa JF, Bauer J. Avaliação da intensidade de luz dos fotopolimerizadores utilizados no curso de odontologia da Universidade Federal do Maranhão. Rev Ciênc Saúde. 2011; 13(1): 26-33.

21. Contarim CR, Casalli JL, Rigo L. Avaliação da potência dos aparelhos fotopolimerizadores utilizados no curso de odontologia da IMED. J Oral Invest. 2015; 4(1): 1925.

22. Freitas SAA, Costa JFC, Bauer JR. O. Avaliação da intensidade da luz dos aparelhos fotopolimerizadores utilizados em clínicas odontológicas de São Luís - MA. Rev Pesq Saúde. 2011;12(2): 27-31.

23. Hao X, Luo M, Wu J, Hu S. A survey of power density of light-curing units used in private dental offices in Changchun City, China. Lasers Med Sci. 2015; 30(2): 493-7.

24. Baldi RL, Teider LD, Leite TM, Martins R, Delgado LAC, Pereira SK. Intensidade de Luz de Aparelhos Fotopolimerizadores utilizados no curso de Odontologia da Universidade Estadual de Ponta Grossa. Publ. UEPG Ci Biol Saúde. 2005; 11(1): 39-46.

25. Alshaafi MM. Evaluation of light-curing units in rural and urban areas. Saudi Dent. 2012: 24(3-4): 163-7.

26. Maghaireh GA, Alzraikat H, Taha N. A. Assessing the irradiance delivered from light-curing units in private dental offices in Jordan. J Am Dent Assoc. 2013; 144(8): 922-7.

27. Wunderlich-Junior AE, Siqueira DF, Scanavini MA, Kanashiro LK. Avaliação de potência de fotopolimerizadores à LED utilizados em consultórios. Ortodontia. 2009;42(2): 95-100.
28. Santos GC, Santos MJ, El-Mowafy O, El-Badrawy W. Intensity of Quart-tungsten-Halogen light polymerization units used in dental offices in Brazil. The International Journal of Prosthodontics. 2005; 18(5): 434- 5.

29. Ribeiro RAO, Lima FFC, Lima IM, Nascimento ABL, Teixeira HM et al. Avaliação da intensidade de luz e da manutenção dos aparelhos fotopolimerizadores utilizados em clínicas odontológicas da cidade do Recife-PE. Rev Odontol UNESP. 2016; 45(6): 351-5.

30. Omidi BR, Gosili A, Jaber-Ansari M, Mahdkhah A. Intensity output and effectiveness of light curing units in dental offices. JClin Exp Dent. 2018;10(6):e555-e560.

31. El-Mowafy O, El-Badrawy W, Lewis DW, et al. Intensity of quartz-tungsten-halogen light-curing units used in private practice in Toronto. J Am Dent Assoc. 2005;136(6):766-73.

32. Hegde V, Jadhav S, Aher GB. A clinical survey of the output intensity of 200 light curing units in dental offices across Maharashtra. J Conserv Dent 2009; 12: 105-108.

33. Federlin M, Price R. Improving light-curing instruction in dental school. J Dent Educ. 2013;77(6):764-72.

34. De Souza GM, El-Badrawy W, Tam LE. Effect of Training Method on Dental Students' Light-Curing Performance. J Dent Educ. 2018;82(8):864-71.

Recibido : 15-07-2020

Aceptado : 12-12-2020 